\title{
Supernova rates in Abell galaxy clusters and implications for metallicity
}

\author{
Avishay Gal-Yam ${ }^{1}$ and Dan Maoz ${ }^{1}$ \\ ${ }^{1}$ School of Physics and Astronomy and Wise Observatory, Tel Aviv University, Tel Aviv 69978, \\ Israel
}

\begin{abstract}
Supernovae (SNe) play a critical role in the metal enrichment of the intracluster medium (ICM) in galaxy clusters. Not only are SNe the main source for metals, but they may also supply the energy to eject enriched gas from galaxies by winds. However, measurements of SN rates in galaxy clusters have not been published to date. We have initiated a program to find SNe in 163 medium-redshift $(0.06<z<0.2)$ Abell clusters, using the Wise Observatory $1 \mathrm{~m}$ telescope. We report here our on first results and describe our main scientific goals. Following the discovery of a SN in the remote periphery $(78 \mathrm{Kpc})$ of the $\mathrm{cD}$ galaxy of Abell 403, we discuss a novel explanation for the centrally enhanced metal abundances indicated by X-ray observations of galaxy clusters.
\end{abstract}

\section{THE PROJECT AND MAIN SCIENTIFIC OBJECTIVES}

We have used the Wise Observatory $1 \mathrm{~m}$ telescope to monitor monthly a sample of 163 rich (richness class $R>0$ ) Abell galaxy clusters, with medium redshift $(0.06<z<0.2)$ northern declination $(\delta>0)$ and small angular size $\left(r<20^{\prime}\right)$. We have also observed "blank" flanking fields for a sub-sample of the clusters. These will be used to study the cluster vs. field SN rates, and to estimate the luminosity contributed by the cluster in each field. We have used unfiltered ("clear") observations to achieve maximum sensitivity, and have a characteristic limiting magnitude of $R \sim 22$. Variable objects are discovered by image subtraction. New subtraction methods have been developed for use in this project (see fig. 1).

Our main scientific goal is to derive from our data the SN rate as a function of various parameters such as host galaxy type and cluster environment: position within the cluster, cluster richness and cluster vs. field. SN rates can then be used to determine the current and past star formation rates in galaxy clusters [1]. Our measured SN rates can replace the assumed rates used so far in studies of metal abundances in the intracluster gas. We also intend to study the rate, distribution and properties of intergalactic SNe in galaxy clusters. A candidate intergalactic SN we have discovered, SN 1998fc (see fig. 3), will be discussed below. Our search is 


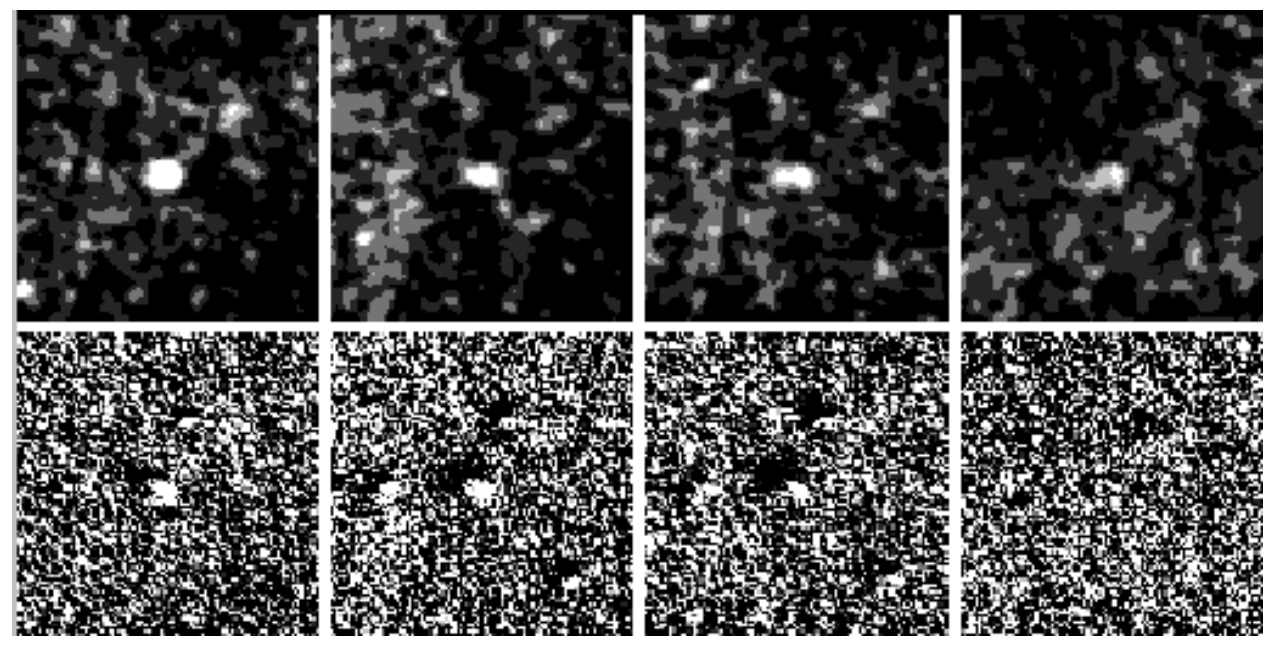

FIGURE 1. Difference images of SN1999ay 1,3,4 and 5 months after discovery produced with our new image subtraction method (upper panel) and with the standard one (lower panel). Note that with the new method this $\mathrm{SN}$ is still detectable after 5 months (upper right)

also sensitive to other optical transients, such as AGNs in the clusters and behind them, flares from tidal disruption of stars by dormant massive black holes in galactic nuclei and GRB afterglows. We may also detect the gravitational lensing effect of the clusters on background $\mathrm{SNe}[2]$.

\section{FIRST RESULTS}

Our program has already discovered 11 spectroscopically confirmed SNe at $z=$ $0.1-0.24$, (see table 1 and fig. 2) and several unconfirmed SNe. We have also detected variable stellar objects (some of which are AGN) and dozens of asteroids.

TABLE 1. Spectroscopically confirmed SNe discovered at the Wise Observatory

\begin{tabular}{|c|c|c|c|c|c|c|c|}
\hline $\mathrm{SN}$ & Cluster & Cluster z & Type & $\mathrm{SN}$ z & Discovered & $\mathrm{R}$ mag & spectrum \\
\hline $1998 \mathrm{cg}$ & Abell 1514 & 0.199 & $\mathrm{Ia}$ & 0.12 & 1.5 .98 & 18.5 & ESO $3.6 \mathrm{~m}$ \\
\hline $1998 \mathrm{eu}$ & Abell 125 & 0.188 & Ia & 0.181 & $14,11.98$ & 19.7 & $\mathrm{AAT} 4 \mathrm{~m}$ \\
\hline $1998 \mathrm{fc}$ & Abell 403 & 0.103 & Ia & 0.10 & 20.12 .98 & 20.5 & ESO 3.6m \\
\hline 1998fd & Field & & Ia & 0.24 & 24.12 .98 & 21.3 & Keck II 10m \\
\hline 1999C & Abell 914 & 0.195 & Ia & 0.125 & 14.1 .99 & 19.6 & Keck II 10m \\
\hline 1999ax & Abell 1852 & 0.181 & Ia & 0.09 & 20.3.99 & 18.5 & $\mathrm{KPNO} 4 \mathrm{~m}$ \\
\hline 1999ay & Abell 1966 & 0.151 & II? & 0.15 & 21.3.99 & 18.0 & $\mathrm{KPNO} 4 \mathrm{~m}$ \\
\hline $1999 \mathrm{cg}$ & Abell 1607 & 0.136 & Ia & 0.14 & 15.4 .99 & 19.2 & Keck II 10m \\
\hline $1999 \mathrm{ch}$ & Abell 2235 & 0.151 & $\mathrm{Ia}$ & 0.15 & 13.5 .99 & 19.8 & $\mathrm{KPNO} 4 \mathrm{~m}$ \\
\hline 1999ci & Abell 1984 & 0.124 & Ia & 0.12 & 15.5.99 & 19.3 & $\mathrm{KPNO} 4 \mathrm{~m}$ \\
\hline $1999 \mathrm{ct}$ & Abell 1697 & 0.183 & $\mathrm{Ia}$ & 0.18 & 13.6 .99 & 20.8 & Keck II 10m \\
\hline
\end{tabular}




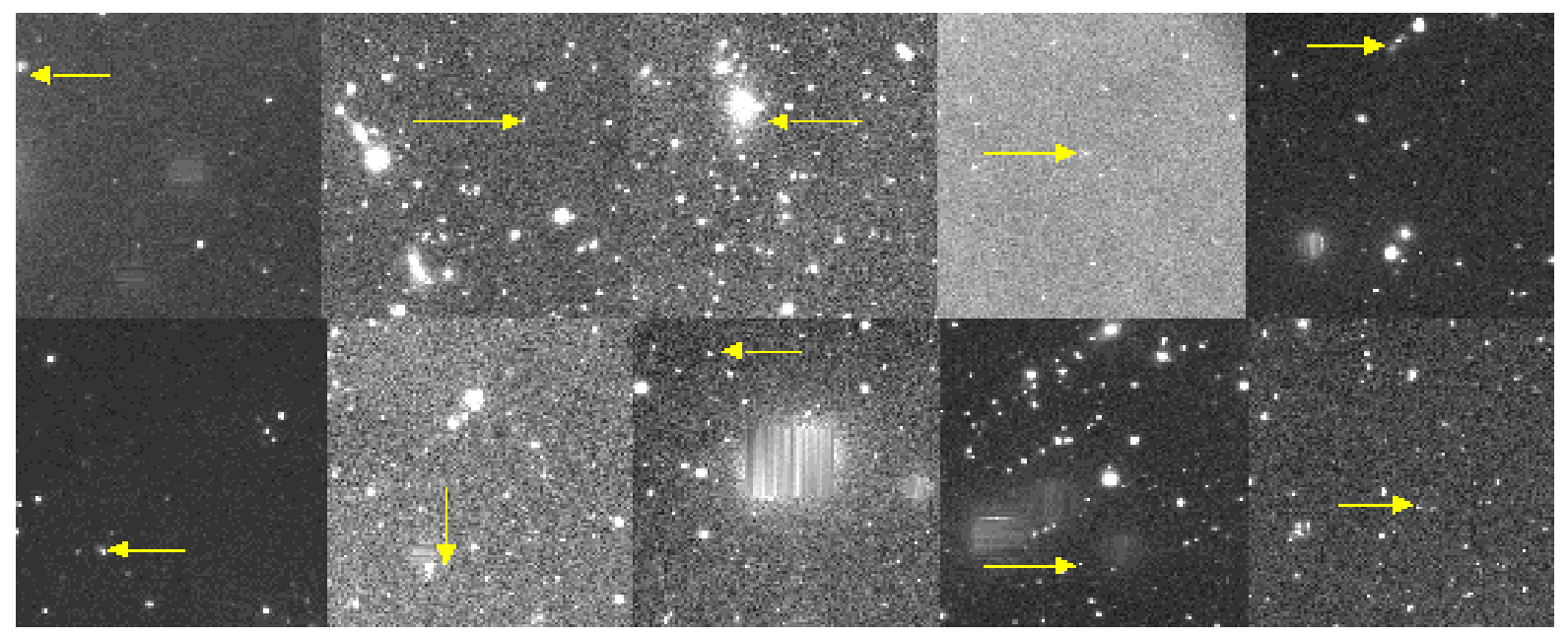

FIGURE 2. A sample of SN images discovered at the Wise Observatory:

(From left to right and top to bottom) SN 1998cg, 1998eu, 1998fc, 1998fd, 1999C, 1999ax, 1999ay, $1999 \mathrm{cg}, 1999 \mathrm{ch}, 1999 \mathrm{ci}$

\section{INTERGALACTIC SNE AND ENHANCED CENTRAL METAL ABUNDANCES IN CLUSTERS}

The existence of a diffuse population of intergalactic stars is supported by a growing body of observational evidence such as intergalactic planetary nebulae in the Fornax and Virgo clusters [3] [4] [5] [6], and intergalactic red giant stars in Virgo [7]. Recent imaging of the Coma cluster reveals low surface brightness emission from a diffuse population of stars [8], the origin of which is attributed to galaxy disruption [9] [10]. Since type Ia SNe are known to occur in all environments, there is no obvious reason to assume that such events do not happen within the intergalactic stellar population. SN 1998fc may be such an event. The intergalactic stellar population is centrally distributed [11]. Therefore, metals produced by intergalactic Ia SNe can provide an elegant explanation for the central enhancement of metal abundances with type Ia characteristics, recently detected in galaxy clusters [12].

\section{SN 1998fc - An intergalactic SN candidate in Abell 403}

SN 1998fc was detected near the cD galaxy of Abell 403 [13], and was spectroscopically confirmed as a type Ia SN at the cluster redshift [14] [15]. The most likely host for this SN, the cD galaxy, is very distant - at least $78 \mathrm{Kpc}$ away. This may be an intergalactic SN whose progenitor star was a member of the diffuse intergalactic stellar population. Alternatively, the host may be a faint dwarf galaxy. The distribution of "hostless" SNe is expected to be different if the progenitors are members of the intergalactic population, centered near the cluster core [11], or members of dwarf galaxies, more abundant in the outskirts of galaxy clusters [16]. Therefore, 


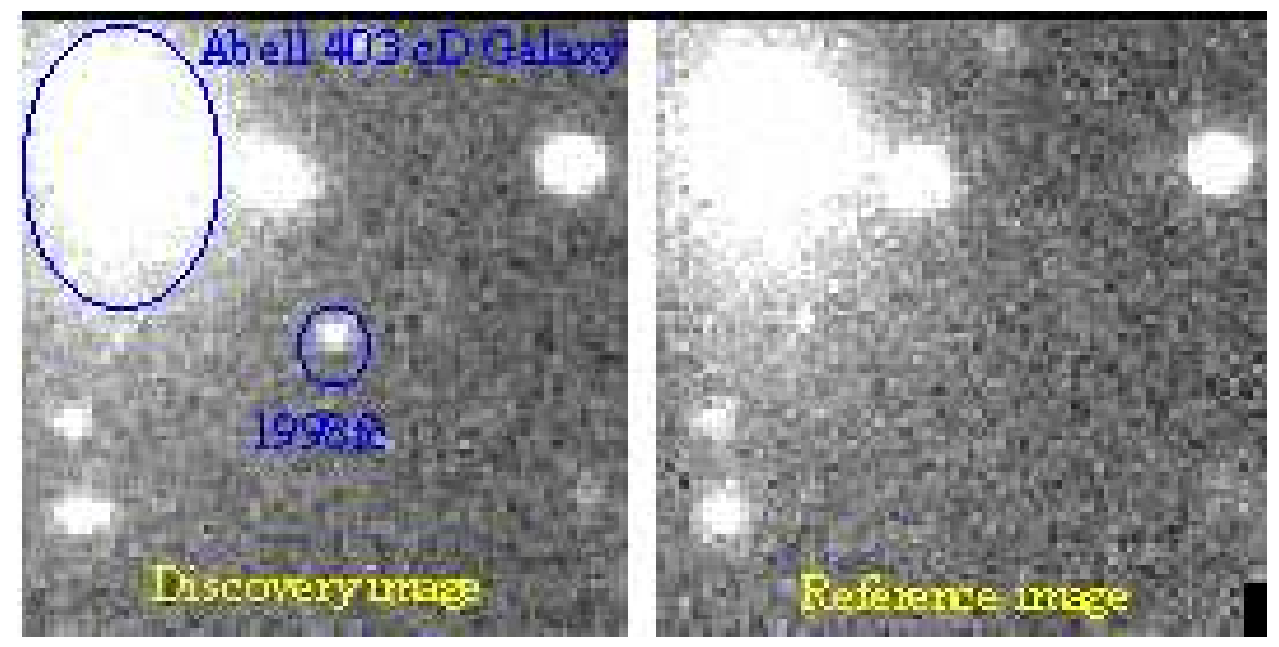

FIGURE 3. SN 1998fc - An intergalactic SN candidate in Abell 403.

the nature of such objects could be resolved with larger number statistics. In any event, the number of SNe with undetected hosts relative to the total number of cluster SNe can put an upper limit on the intergalactic stellar fraction.

\section{REFERENCES}

1. Madau, P., DellaValle, M., \& Panagia, N. 1998, MNRAS, 297, L17

2. Kolatt, T., \& Bartelmann, M. 1998, MNRAS, 296, 763

3. Theuns, T., \& Warren, S.J. 1996, MNRAS, 284, L11

4. Arnaboldi, M. et al. 1996, ApJ, 472, 145

5. Ciardullo, R. et al. 1998, ApJ, 492, 62

6. Freeman, K. C. et al. 1999, astro-ph/9910057

7. Ferguson, H.C., Tanvir, N.R., \& von Hippel, T. 1998, Nature, 391, 461

8. Gregg, M.D. \& West, M.J., 1998, Nature, 396, 549

9. Dubinski, J., Mihos, J.C. \& Hernquist, L., 1996, ApJ, 462, 576

10. Moore, B., et al. 1996, Nature, 379, 613

11. Dubinski, J., 1999, astro-ph/9902331

12. Dupke, R.A. \& White, R.E.III, 1999, ApJ, submitted, astro-ph/9902112

13. Gal-Yam, A. \& Maoz, D., 1999, IAUC 7082

14. Gal-Yam, A. \& Maoz, D., 1999, IAUC 7093

15. Filippenko, A.V., et al. 1999, IAUC 7091

16. Phillipps, S., Driver, S., Couch, W. J. \& Smith, R. M., 1998, ApJ, 498, 119 